\title{
Image Transmission in Sensor Networks
}

\author{
King-Shan Lui and Edmund Y. Lam \\ Department of Electrical and Electronic Engineering \\ The University of Hong Kong \\ Pokfulam Road, Hong Kong, China \\ Email: \{kslui,elam\}@eee.hku.hk
}

\begin{abstract}
Wireless sensor networks allow fine-grained monitoring of the environment. However, as sensors have physical limitations in energy, processing power, and memory, etc., techniques have to be developed to efficiently utilize the limited resource available in a sensor network. In this paper, we study the image tranmission problem in sensor networks. Cameras are installed in various locations of a wide area to take images of targeted objects. These images have to be sent back to a centralized server, which may be very far away from the cameras. Therefore, the images have to traverse the sensors hop by hop to the server. As images usually contain a large amount of data, if they are sent individually, the communication overheads will be huge. To reduce the overheads, we can pre-process the images in the sensors before sending them back to the server, but this preprocessing requires extra energy in the sensors. In this paper, we study how images can be efficiently transmitted through a sensor network. We aim at reducing the energy needed in transmitting the images while maintaining the quality of the combined image.
\end{abstract}

\section{INTRODUCTION}

A wireless sensor network is a network consisting of thousands of sensors that span a large geographical region. These sensors are able to communicate with each other to collaboratively detect objects, collect information, and transmit messages. Sensor networks have become an important technology especially for environmental monitoring, military applications, disaster management, etc [1] [2]. However, as sensors are usually small in size, they have many physical limitations. For example, due to its limited size, a sensor does not have a very powerful CPU and is limited in computational power and memory. On the other hand, a sensor is powered by a battery instead of a power outlet. This limitation in energy puts extra constraints in the operations of sensors. As recharging is difficult, sensors should smartly utilize its limited energy in collecting, processing, and transmitting information.

In this paper, we investigate the problems and issues of sensor network cameras. Cameras are installed in various locations of a wide area sensor network to monitor and track objects. Each camera is connected to a sensor and takes images. These images may overlap with each other. The sensor can process the images taken by the camera, but only in a limited manner. More importantly, the sensor, which is equipped with communication devices, is responsible for sending these images to a centralized server for more sophisticated processing. As the centralized server may be very far away from the cameras, the images then have to be transmitted hop by hop through the sensor network to the server. This requires energy in the sensors and the amount of energy needed for sending one image depends on the size of the image and the number of hops that the image has to pass through. The size of images can be reduced by combining images with overlapping regions. Different paths may have different hop counts. In this paper, we investigate how to optimize the energy resources in transmitting images through selecting appropriate paths and appropriate compression of images.

In general, a sensor node can reduce the energy needed in transmission by combining the data it receives from several neighbors together before sending it out. This idea is called data aggregation and the problem of finding optimal data aggregation is NP-hard [3]. Most of the work [4] [5] [6] focus on aggregating simple scalar data such as averages, maxima, and minima. Only a few of them study the employment of aggregation in image transmission. In [7], the authors show that applying maximum compression before transmission may not always entail the minimal amount of energy. The authors develop a heuristic to determine a good compression level. Distributed image compression is studied in [8]. The whole process of compression is distributed among different groups of sensor nodes. By using this approach, the total energy needed is increased but the maximum energy needed in a sensor node is reduced and the lifetime of sensor nodes can be prolonged. [9] also studies distributed image compression. Overlapping areas of images are identified and sensors send a low-resolution version of these areas for the receiver to reconstruct the overlapped blocks in high-resolution. None of the work mentioned above consider routing issues in image transmission. SPIN-IT [10] is a routing protocol for retrieving an image based on metadata of images. It focuses on how a node identifies the sources of an image requested but not the image transmission issues.

In this paper, we study the problem of selecting forwarding paths for sending images. We first demonstrate that the amounts of energy required in different forwarding paths are different. We then develop an algorithm for a sensor node to select a path that requires the least energy. Finally, we study the performance of our algorithm using simulations. The rest of paper is organized as follows: Section II presents the network model and problem statement. Section III describes our algorithm and simulation results are shown in Section IV. We finally conclude our paper in Section V. 


\section{Problem Statement}

\section{A. Network Model}

Figure 1 depicts the network model adopted in this paper. There are $N$ camera-equipped sensors and they are nodes $C_{1}$ to $C_{N}$. They all take images of the same object but from different perspectives. The images should be sent back to the server $S$, which is several hops away from the cameras. Sensors $P_{1}$ to $P_{N-1}$ are nodes that are adjacent to the camera sensors. Apart from transmitting images, these sensors are able to perform image processing functions. Node $P_{i}$ is a direct neighbor of sensors $C_{i}$ and $C_{i+1}$. This relationship is reflected by the lines connecting $P_{i}$ to $C_{i}$ and $C_{i+1} \cdot C_{i}$ and $C_{i+1}$ are also direct neighbors. Therefore, $C_{i}$ can send its images directly to $C_{i-1}, C_{i+1}$, or $P_{i}$. Nodes $C_{i}$ and $P_{i}$ can combine the images they receive and send the combined image out. Energy may be saved in this process and the details will be discussed in the next section. The images sent by $P_{i}$ will go hop by hop to server $S$. The paths are represented as wavy arrows in the figure. To reduce the cost of deployment, the intermediate nodes on the path from $P_{i}$ to $S$ are equipped with communication devices only. They do not have image processing functions and cannot combine images they receive.

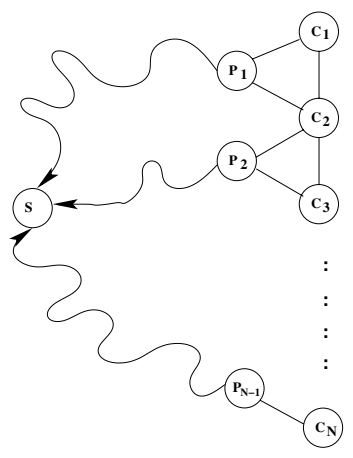

Fig. 1. Network Model

\section{B. Image Transmission}

In this section, we show that the energy needed may be different if we transmit images using different paths. To simplify our discussion, we illustrate the idea using the transmission of the images captured by cameras $C_{1}, C_{2}$, and $C_{3}$ only. The notations used are defined in Table I.

We assume that JPEG is used for compressing images. To simplify our analysis, we further assume that the sizes of raw images captured by the cameras are the same and the compression ratios are roughly constant. To reduce noise, every camera has to send the entire image to $S$ or other nodes for processing even though images may be overlapped with each other. For example, suppose that $I_{1}$ and $I_{2}$ overlap with each other in region $I_{1} \cap I_{2}$. When $P_{1}$ receives these two images, it can stitch them to form $I_{1+2}$ [11]. In the current work, we assume that the duplicate information in $I_{1} \cap I_{2}$ will be averaged to reduce noise, and that the computational cost to perform the averaging is negligible compared to the energy

\begin{tabular}{|c||l|}
\hline Notation & Meaning \\
\hline \hline$I_{i}$ & Compressed Image captured by $C_{i}$ \\
\hline$|I|$ & Data size of image $I$ after compression \\
\hline$I_{a+b}$ & Combined image of $I_{a}$ and $I_{b}$ \\
\hline$c(s)$ & $\begin{array}{l}\text { Energy needed to compress an image such that the } \\
\text { resultant data size is } s\end{array}$ \\
\hline$d(s)$ & $\begin{array}{l}\text { Energy needed to decompress a compressed image of } \\
\text { data size } s\end{array}$ \\
\hline$t(s)$ & Energy needed to send data size $s$ to a neighbor sensor \\
\hline$h\left(P_{i}\right)$ & Number of hops on the path from $P_{i}$ to $S$ \\
\hline
\end{tabular}

TABLE I

NOTATIONS

used in other processes such as transmission or compression. Therefore, for the stitching to be possible, the overlapping regions of $I_{1}$ and $I_{2}$ have to be decompressed, processed, and recompressed to form a new version of that region. Although $\left|I_{1}\right|,\left|I_{2}\right|$, and $\left|I_{3}\right|$ are of the same size, $\left|I_{1} \cap I_{2}\right|$ and $\left|I_{2} \cap I_{3}\right|$ may not be the same. Lest a sensor may use up too much energy in transmitting an image, at most two images can be combined together.

Images can be sent and combined in many different ways. Figure 2 shows all possible ways of transmitting images $I_{1}, I_{2}$ and $I_{3}$ to the server through $P_{1}$ and $P_{2}$, with the assumption that a node can combine at most two images.

- Method A: $I_{1}$ and $I_{2}$ are sent to $P_{1}$ while $I_{3}$ is sent to $P_{2}$. Upon receiving $I_{1}$ and $I_{2}, P_{1}$ combines $I_{1}$ and $I_{2}$ to form $I_{1+2}$ and sends it out.

- Method B: $I_{1}$ is sent to $P_{1}$ while $I_{2}$ and $I_{3}$ are sent to $P_{2}$. Upon receiving $I_{2}$ and $I_{3}, P_{2}$ combines them to form $I_{2+3}$ and sends it out.

- Method C: $C_{2}$ sends $I_{2}$ to $C_{1}$ to form $I_{1+2}$. $C_{1}$ then sends $I_{1+2}$ through $P_{1}$ and $I_{3}$ is sent to $P_{2}$.

- Method D: $C_{1}$ sends $I_{1}$ to $C_{2}$ to form $I_{1+2}$. $C_{2}$ then sends $I_{1+2}$ through $P_{1}$ and $I_{3}$ is sent to $P_{2}$.

- Method E: $C_{2}$ sends $I_{2}$ to $C_{3}$ to form $I_{2+3}$. $C_{3}$ then sends $I_{2+3}$ through $P_{2}$ and $I_{1}$ is sent to $P_{1}$.

- Method F: $C_{3}$ sends $I_{3}$ to $C_{2}$ to form $I_{2+3}$. $C_{2}$ then sends $I_{2+3}$ through $P_{2}$ and $I_{1}$ is sent to $P_{1}$.

We now analyze the energy needed in these methods. Both image transmission and image processing require energy. The amount needed in transmission depends on the size of the image and the number of hops that it traverses. We assume that the energy needed to traverse each hop is the same for the same image. Therefore, the total energy needed to send image $I$ from $P_{1}$ to $S$ is $h\left(P_{1}\right) * t(|I|)$. The energy needed to produce $I_{1+2}$ depends on the size of $I_{1} \cap I_{2}$ and where the combination process is carried out. For example, in Method A, $P_{1}$ combines $I_{1}$ and $I_{2}$. To combine the region $I_{1} \cap I_{2}$ in 


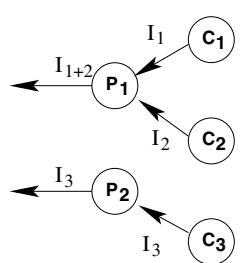

Method A

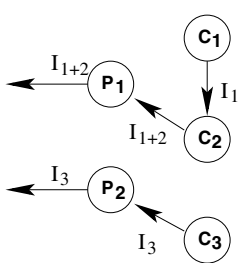

Method D

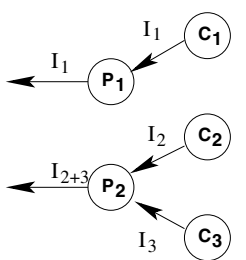

Method B

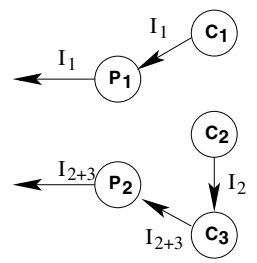

Method E

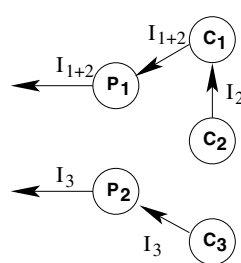

Method C

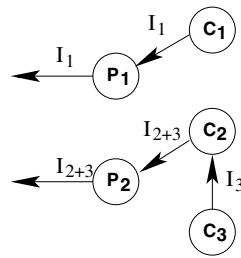

Method F
Fig. 2. Six different ways of transmission

$I_{1}$ and $I_{2}, P_{1}$ has to first decompress the overlapping portion in each image and then recombine them. The non-overlapping regions in both images remain untouched. The energy needed in decompression is $2 * d\left(\left|I_{1} \cap I_{2}\right|\right)$ while the energy needed in compression is $c\left(\left|I_{1} \cap I_{2}\right|\right)$. As a result, in forming $I_{1+2}, P_{1}$ in Method A needs $2 * d\left(\left|I_{1} \cap I_{2}\right|\right)+c\left(\left|I_{1} \cap I_{2}\right|\right)$ amount of energy.

On the other hand, the decompression and compression are carried out in $C_{1}$ in Method $\mathrm{C}$. To form $I_{1} \cap I_{2}$ upon receiving $I_{2}, C_{1}$ only needs to decompress the overlapping portion of $I_{2}$. It does not have to decompress $I_{1}$ since it has the raw image of $I_{1}$. The energy needed in getting $I_{1} \cap I_{2}$ is thus $d\left(\mid I_{1} \cap\right.$ $\left.I_{2} \mid\right)+c\left(\left|I_{1} \cap I_{2}\right|\right)$. The non-overlapping region of $I_{2}$ remains untouched and no energy is needed to process that region. However, $C_{1}$ has to produce the non-overlapping region of $I_{1}$ and the energy required is $c\left(\left|I_{1}\right|-\left|I_{1} \cap I_{2}\right|\right)$, which is $c\left(\left|I_{1}\right|\right)-c\left(\left|I_{1} \cap I_{2}\right|\right)$. Then, the total energy needed to produce $I_{1+2}$ is $d\left(\left|I_{1} \cap I_{2}\right|\right)+c\left(\left|I_{1}\right|\right)$.

We now explain the energy needed in Method A. The energy needed in transmission is the sum all energy needed in the "arrows" in figure, which is

$$
t\left(\left|I_{1}\right|\right)+t\left(\left|I_{2}\right|\right)+t\left(\left|I_{3}\right|\right)+h\left(P_{1}\right) * t\left(\left|I_{1+2}\right|\right)+h\left(P_{2}\right) * t\left(\left|I_{3}\right|\right)
$$

As $\left|I_{1}\right|=\left|I_{2}\right|=\left|I_{3}\right|=|I|$, (1) can be simplied to

$$
\left(h\left(P_{2}\right)+3\right) * t(|I|)-h\left(P_{1}\right) * t\left(\left|I_{1+2}\right|\right)
$$

Note that $\left|I_{1+2}\right|=2|I|-\left|I_{1} \cap I_{2}\right|$. (1) can be further simplied to

$$
\left(2 h\left(P_{1}\right)+h\left(P_{2}\right)+3\right) * t(|I|)-h\left(P_{1}\right) * t\left(\left|I_{1} \cap I_{2}\right|\right)
$$

The energy needed in processing is the sum of the energy spent in compression and decompression in each node. In Method A, $C_{1}, C_{2}$, and $C_{3}$ each requires $c(|I|)$ to compress its image. $P_{1}$ needs $2 * d\left(\left|I_{1} \cap I_{2}\right|\right)+c\left(\left|I_{1} \cap I_{2}\right|\right)$ as discussed above. $P_{3}$ does not spend energy in processing $I_{3}$ since it does neither compression nor decompression. The energy needed in processing in Method $\mathrm{A}$ is thus

$$
3 c(|I|)+2 d\left(\left|I_{1} \cap I_{2}\right|\right)+c\left(\left|I_{1} \cap I_{2}\right|\right)
$$

The energy needed for Methods B-F can be calculated in a similar way. We summarize the results as follows. T stands for Transmission and $\mathrm{P}$ stands for Processing.

- Method A

$\mathrm{T}:\left(2 h\left(P_{1}\right)+h\left(P_{2}\right)+3\right) * t(|I|)-h\left(P_{1}\right) * t\left(\left|I_{1} \cap I_{2}\right|\right)$

P: $3 c(|I|)+2 d\left(\left|I_{1} \cap I_{2}\right|\right)+c\left(\left|I_{1} \cap I_{2}\right|\right)$

- Method B

$\mathrm{T}:\left(h\left(P_{1}\right)+2 h\left(P_{2}\right)+3\right) * t(|I|)-h\left(P_{2}\right) * t\left(\left|I_{2} \cap I_{3}\right|\right)$

P: $3 c(|I|)+2 d\left(\left|I_{2} \cap I_{3}\right|\right)+c\left(\left|I_{2} \cap I_{3}\right|\right)$

- Method C and Method D

$\mathrm{T}:\left(2 h\left(P_{1}\right)+h\left(P_{2}\right)+4\right) * t(|I|)-\left(h\left(P_{1}\right)+1\right) * t\left(\left|I_{1} \cap I_{2}\right|\right)$ P: $3 c(|I|)+d\left(\left|I_{1} \cap I_{2}\right|\right)$

- Method E and Method F

$\mathrm{T}:\left(h\left(P_{1}\right)+2 h\left(P_{2}\right)+4\right) * t(|I|)-\left(h\left(P_{2}\right)+1\right) * t\left(\left|I_{2} \cap I_{3}\right|\right)$

P: $3 c(|I|)+d\left(\left|I_{2} \cap I_{3}\right|\right)$

It can be observed that determining which method would use the least amount of energy is not trivial. The problem gets substantially more complicated when there are more cameras. In the next section, we describe an algorithm for nodes $C_{i}$ to determine which method to use in order to reduce the energy needed in sending the images to the server.

\section{ThE Algorithm}

In order to find out which method requires least energy for transmitting $I_{1}, I_{2}$, and $I_{3}$, we need to know $h\left(P_{1}\right), h\left(P_{2}\right)$, $|I|,\left|I_{1} \cap I_{2}\right|$, and $\left|I_{2} \cap I_{3}\right|$. Generally speaking, if a node wants to know which way is the best for transmitting images $I_{1} \ldots I_{k}$, the node has to obtain $h\left(P_{1}\right) \ldots h\left(P_{k-1}\right),|I|$, and $\mid I_{i} \cap$ $I_{i+1} \mid$ for $i=1$ to $k-1$. The larger $k$, the higher overhead in obtaining the information since cameras are farther apart. When $k=3$, just like the situations in Figure 2, the center camera, $C_{2}$, is a neighbor of all other nodes in the group. $C_{2}$ is able to obtain all necessary information in one hop and inform the decision to other nodes in one broadcast message. If $k$ is 4 or larger, no node can be a neighbor of all nodes anymore and information and decision propagation becomes more expensive. On the other hand, the number of possible methods increases exponentially with $k$. To avoid overloading the decision-making node, we should select a small $k$.

We design our algorithm based on these observations and decide to set $k$ to be 3 . The cameras are divided into nonoverlapping groups of 3. Since each camera knows its identifier, it can determine which group it belongs to by itself and whether it is the one in the center. The center camera in a group is responsible for collecting information, making decision, and informing neighbor nodes the decision. Suppose that a group consists of cameras $C_{i-1}, C_{i}$, and $C_{i+1}$. The center camera, $C_{i}$, collects $h\left(P_{i-1}\right)$ from $P_{i-1}, h\left(P_{i}\right)$ from $P_{i}$ and some information about $I_{i-1}$ and $I_{i+1}$ from $C_{i-1}$ 
and $C_{i+1}$, respectively, so that it can compute $\left|I_{i-1} \cap I_{i}\right|$ and $\left|I_{i} \cap I_{i+1}\right|$. Based on the information, $C_{i}$ can decide which transmission method is the best. $C_{i}$ then informs other nodes by sending a single broadcast message. When $C_{i-1}$ and $C_{i+1}$ receives the message, they can send their images according to the method selected.

There is overhead involved in exchanging information and decision. The algorithm is not justified if the overhead is large. We now argue that the overhead we need is negligible. It is not difficult to see that $|I|, h\left(P_{i-1}\right)$, and $h\left(P_{i}\right)$ are integers. They are very small in size. In addition, these three values do not change often. $C_{i}$ does not need to ask for them every time it processes a new image. Instead, $P_{i-1}$ and $P_{i}$ only need to inform $C_{i}$ when the values change. Therefore, the overhead in getting hop count information is very small.

Another piece of information $C_{i}$ needs is the size of $\mid I_{i-1} \cap$ $I_{i} \mid . C_{i}$ uses the orientations of $I_{i-1}$ and $I_{i}$ to find out the overlapping region and the size. That is, $C_{i-1}$ only has to send the orientation information of its image to $C_{i}$. Again, the size of this information is small and can be neglected.

\section{Simulation}

In this section, we simulate various scenarios where $C_{2}$ would pick different transmission methods based on the information it receives, for a group consisting of $C_{1}, C_{2}$, and $C_{3}$. Without loss of generality, we only consider cases where $\left|I_{1} \cap I_{2}\right| \leq\left|I_{2} \cap I_{3}\right|$. Depending on the applications, the energy consumed in transmitting versus compressing or decompressing the same amount of image data can vary substantially [7]. Therefore, in each scenario, we examine the transmission energy required for different methods under various ratios of transmission energy to compression energy. Compression and decompression are assumed to take the same amount of energy under JPEG.

In the first case, we assume $h\left(P_{1}\right)=h\left(P_{2}\right)=1$ and $\left|I_{1} \cap I_{2}\right|=\left|I_{2} \cap I_{3}\right|=0.3|I|$, i.e. $30 \%$ overlap between the images. The energy consumption is shown in figure 3 . It is not surprising that when transmission only accounts for a fraction of the cost of compression, methods $\mathrm{C}, \mathrm{D}, \mathrm{E}$, and $\mathrm{F}$ consume less energy because they require less energy for compression. On the other hand, when transmission cost is high, methods A and $\mathrm{B}$ are preferable because the total path length is shorter. The cross-over point, though, is when transmission energy equals about $86 \%$ of the compression energy, so there is a bias to favor strategies with less compression.

In the second case, we still assume $h\left(P_{1}\right)=h\left(P_{2}\right)=1$, but $\left|I_{1} \cap I_{2}\right|=0.3|I|$ and $\left|I_{2} \cap I_{3}\right|=0.7|I|$. The plots for small transmission energy and large transmission energy are shown in figure 4(a) and (b), respectively. Three strategies are possible: When the transmission energy is large, method $\mathrm{B}$ is preferred because it requires the least transmission. When transmission and compression energies are comparable, methods $\mathrm{E}$ and $\mathrm{F}$ provide the best balance and least energy as a result. However, when transmission energy is relatively very small, methods $\mathrm{C}$ and $\mathrm{D}$ are preferred. This is apparently counter-intuitive, because $C_{2}$ would rather stitch with $C_{1}$ to

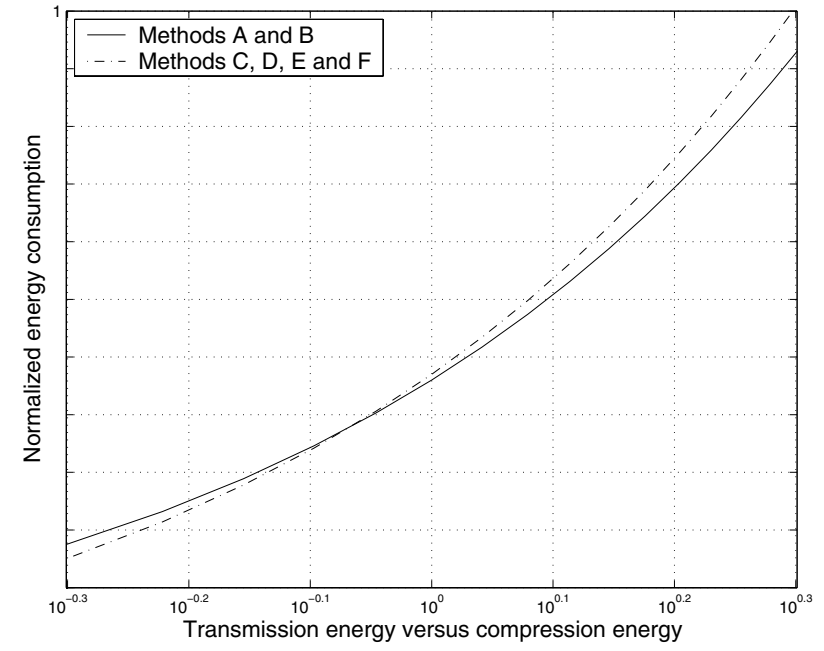

Fig. 3. Case I: equal path length and overlap.

which it has a smaller overlap. We can however explain this by noting that these methods require less compression and decompression due to the smaller amount of overlap.

In the third case, we assume $h\left(P_{1}\right)=1$ but $h\left(P_{2}\right)=2$, and the amount of overlap the same as case 2 above. The situation for low transmission energy is the same as above. However, for moderate to high transmission energy, method A would be preferred: even though $C_{2}$ has closer resemblance to $C_{3}$, because the path is shorter via $P_{1}$, it would rather stitch with $C_{1}$ and transverse on a shorter path.

\section{CONCLUSION}

In this paper, we have investigated the transmission of images captured in a network of cameras. We show that when there is overlap in the scene captured and intermediate image stitching is possible, the image transmission strategy is nontrivial. Under different ratios of transmission and compression energy, scene overlap, and network path length, we should perform different routing of the images. Algorithm and simulations are provided for the situation with three cameras to find the suitable routing strategy under different cases.

\section{REFERENCES}

[1] D. Estrin, D. Culler, K. Pister, and G. Sukhatme, "Connecting the physical world with pervasive networks," in IEEE Pervasive Computing, 2002, pp. $59-69$.

[2] G. J. Pottie and W. J. Kaiser, "Wireless integrated network sensors," in Communications of the ACM, 2000, vol. 43, pp. 51 - 58.

[3] B. Krishnamachari, D. Estrin, and S. Wicker, "The impact of data aggregation in wireless sensor networks," in International Workshop of Distributed Event Based Systems, 2002, pp. 575 - 578.

[4] M. Ding, X. Cheng, and G. Xue, "Aggregation tree construction in sensor networks," in IEEE Vehicular Technology Conference, 2003, pp. $2168-2172$.

[5] N. Shrivastava, C. Buragohain, and D. Agrawal, "Medians and beyond: New aggregation techniques for sensor networks," in ACM SenSys, 2004.

[6] S. Nath, P. B. Gibbons, S. Seshan, and Z. R. Anderson, "Synopsis diffusion for robust aggregation in sensor networks," in ACM SenSys, 2004. 


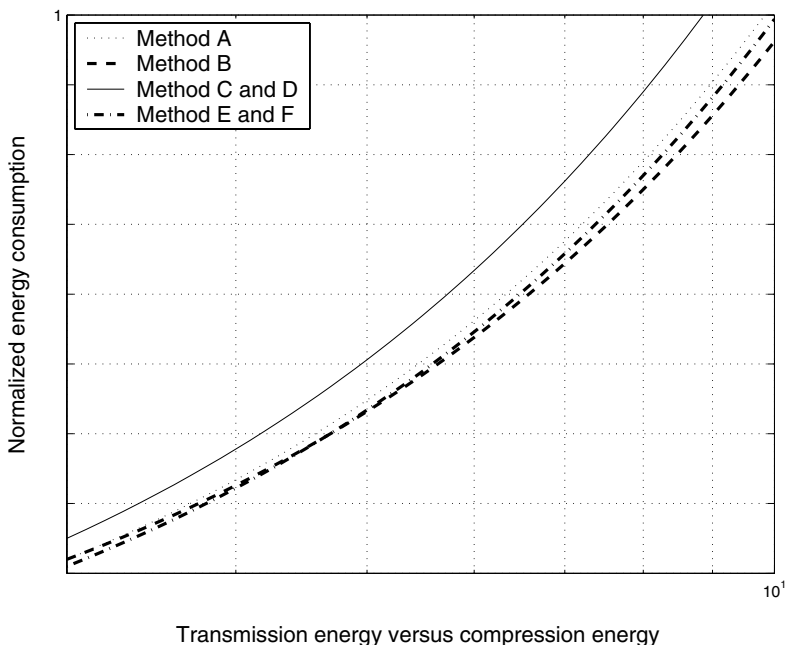

(a) Large transmission energy

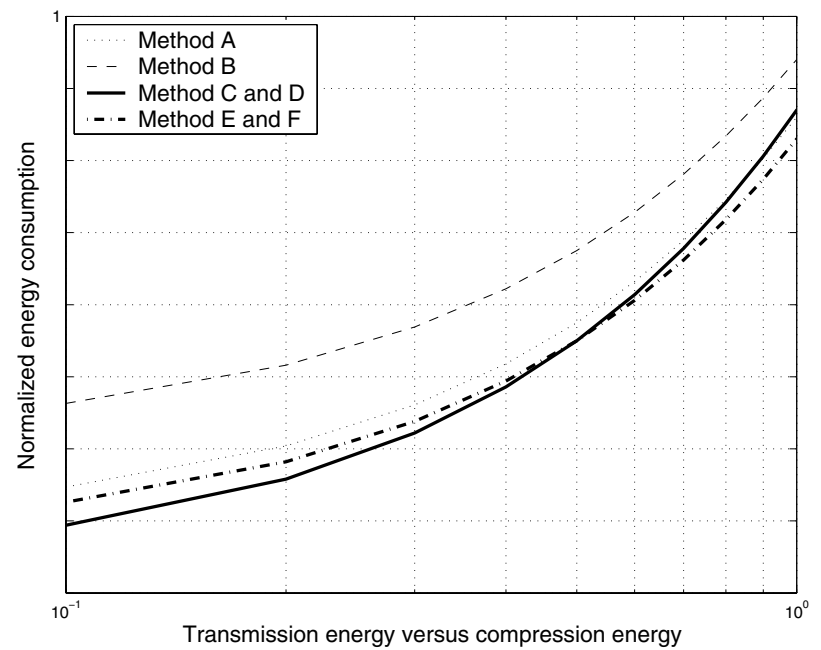

(b) Small transmission energy

Fig. 4. Case II: equal path length and unequal overlap.

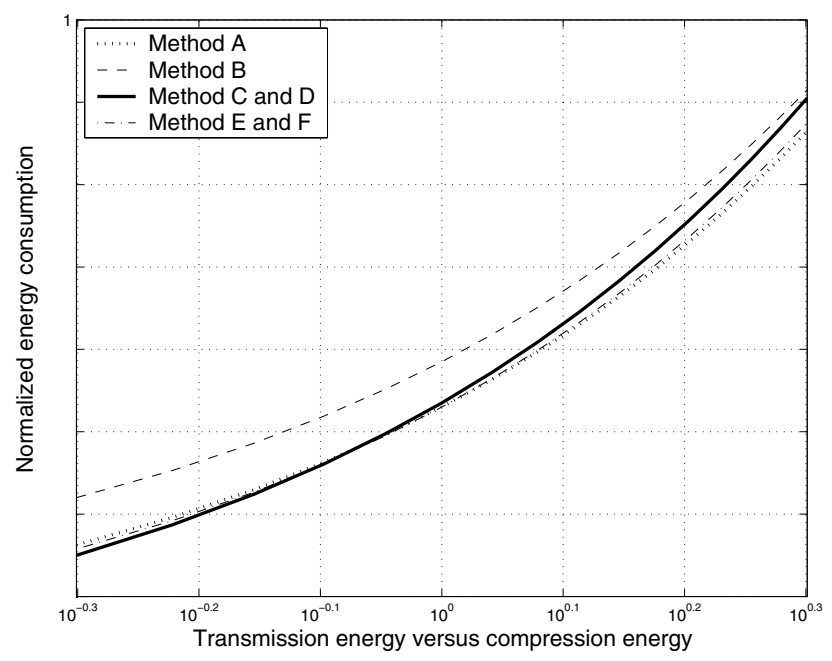

Fig. 5. Case III: unequal path length and overlap.
[7] H. Wu and A. A. Abouzeid, "Power aware image transmission in energy constrained wireless networks," in IEEE Symposium on Computers and Communications (ISCC), 2004, pp. $202-207$.

[8] H. Wu and A. A. Abouzeid, "Energy efficient distributed JPEG2000 image compression in multihop wireless networks," in Workshop on Applications and Services in Wireless Networks (ASWN), 2004.

[9] R. Wagner, R. Nowak, and R. Baraniuk, "Distributed image compression for sensor networks using correspondence analysis and super-resolution," in IEEE International Conference on Image Processing, 2003, pp. 597 -600 .

[10] E. Woodrow and W. Heinzelman, "SPIN-IT: A data centric routing protocol for image retrieval in wireless networks," in IEEE International Conference on Image Processing, 2002, pp. 913 - 916.

[11] J. Davis, "Mosaics of scenes with moving objects," in Conference on Computer Vision and Pattern Recognition, 1998, pp. 354 - 360. 